Neurosurg Focus 10 (4):Article 2, 2001, Click here to return to Table of Contents

\title{
Biomechanics of bone fusion
}

\author{
Robert J. Kowalski, M.D., M.S., P.E., LiSA A. Ferrara, M.S., AND \\ EDWARD C. BENZEL, M.D., F.A.C.S.
}

Department of Neurosurgery, Cleveland Clinic Foundation, Cleveland, Ohio

\begin{abstract}
Bone fusion can be achieved by one or more of three methods: in situ, onlay, and interbody fusion. Interbody implants provide the spine with the ability to bear an axial load. They function optimally when placed along the neutral axis and produce little, if any, significant bending moment. Interbody implants may be comprised of bone, nonbone materials such as acrylic, or a combination of both such as in interbody cages.

In this report the authors' goal is to provide some insight into the theoretical, as well as practical, biomechanical factors that influence bone fusion, focusing on interbody implants. They review the concept of stress shielding and its impact on fusion. With the attendant biomechanical nuances of the different regions of the spine, they discuss regionspecific strategies involved in successful fusion. Finally, they review intraoperative techniques that will improve the chance of achieving a successful arthrodesis.
\end{abstract}

KeY WoRdS • biomechanics • spinal fusion • bone graft

Bone fusion may be achieved using one or more of three surgical strategies: in situ, onlay, or interbody fusion. In situ fusions are used when native bone is allowed to come in contact with other native bone that was previously prevented from doing so because of intervening soft tissue. A common clinical example is the denuding of facet cartilage performed in conjunction with placing a lateral mass plate system in the cervical spine. Onlay fusion techniques rely on a decorticated graft bed and the subsequent application of cortical and cancellous autograft. Interbody implants provide the spine with the ability to bear an axial load; they function optimally when placed along the neutral axis and thus produce little, if any, significant bending moment. Interbody implants may be comprised of bone, nonbone materials such as acrylic, or a combination of both, such as interbody cages. All three methods may be used alone or in combination with other implants that can be applied through anterior and/or posterior applications.

The application of interbody implants has more complex biomechanical implications than the other two implants and thus is the focus of this discussion. Interbody implants benefit from the compression-related augmentation of the bone healing-enhancing forces, as predicted by the Wolff law.

The theoretical, as well as practical, biomechanical factors that influence bone fusion are presented here. First, we review of the concept of using bone as an implant material. Second, with the attendant biomechanical nuances of the different regions of the spine, we discuss regionspecific strategies. Third, we review the intraoperative

Abbreviations used in this paper: CSRS $=$ Cervical Spine Research Society; NASS = North American Spine Society; PLIF = posterior lumbar interbody fusion; TIFC $=$ threaded interbody fusion cage; $\mathrm{VB}=$ vertebral body. techniques that will improve the chance of achieving a successful arthrodesis.

\section{BONE AS AN IMPLANT}

Theoretically, there is an optimal distribution of load between a spinal implant and a bone graft. Such ideal balance provides stability, yet allows the graft to "see" the necessary compressive forces for healing, as delineated in the Wolff law. Seventy percent of the load should optimally be applied through the bone graft in both the thoracic and lumbar spine (BC Cheng, CSRS, 1997). When a disproportionate amount of the load is borne by the implant, the chance of achieving a solid arthrodesis diminishes. Stress shielding is defined as an implant induced reduction of bone healing enhancing stresses and loads to such a degree that stress reduction osteoporosis, or nonunion, may result. In addition to the most common culprit-spinal instrumentation-inactivity, such as bedrest, and spinal bracing may also play a role.

In a head-to-head comparison, one group of authors reviewed their clinical experience with four anterior cervical strategies: 1) strut graft combined with halo brace; 2) anterior cervical plate combined with strut graft; 3) anterior strut graft combined with posterior instrumentation and fusion; and 4) anterior strut graft combined with a kickplate; the pseudarthrosis rates were $20 \%, 40 \%, 0 \%$, and 7\%, respectively (ES Doh, CSRS, 1998). Several conclusions may be drawn from these findings. Fusion in which no instrumentation was used in this series was relatively efficacious. This has been borne out in other studies as well (A Hildibrand, NASS, 1997). ${ }^{1025-27}$ The stress shielding of the rigid plate most likely contributed to the high pseudarthrosis rate. The addition of a posterior fusion appears to prevent settling while promoting fusion. 


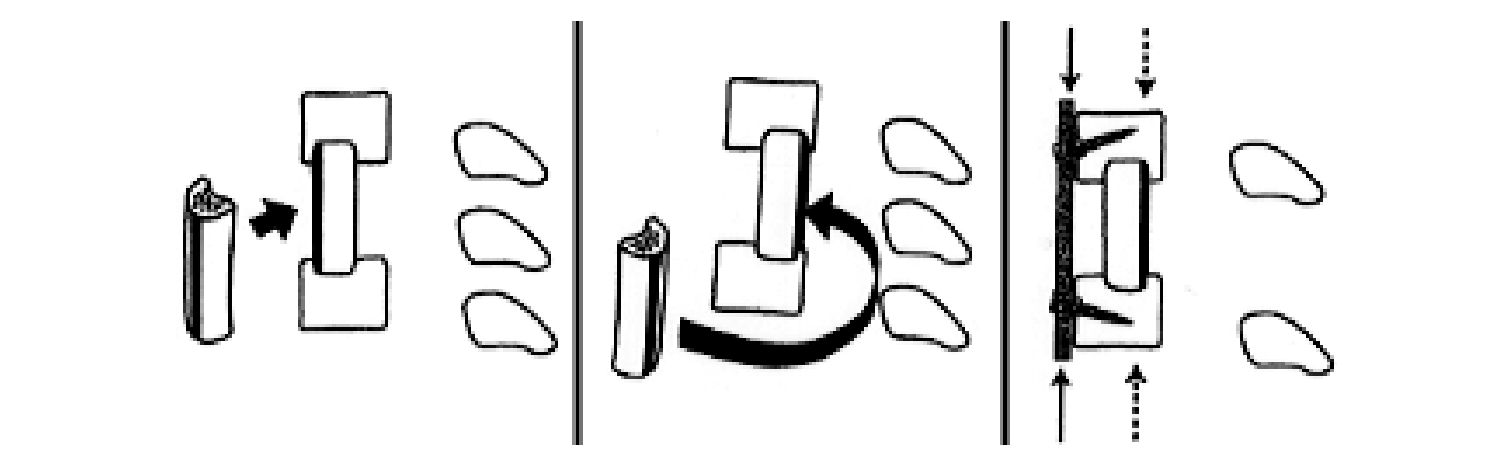

Fig. 1. Left: Placing the crestal portion of a cervical interbody iliac crest graft anteriorly allows it to create an enhanced buttressing effect. Note that the cortical portion of the graft is aligned with the anterior cortex of the VB (arrow). This somewhat enhances the axial load-bearing ability. It significantly enhances the ability to resist kyphosis. Center: If the crestal portion of a cervical interbody iliac crest graft is placed in line with the neutral axis, axial loads are effectively borne, but the prevention of kyphosis is somewhat limited (curved arrow). Right: The placement of the crestal portion of a cervical interbody iliac crest strut graft posteriorly, in combination with an anterior plate (arrows), allows the axial load to be shared between the anterior implant and the posteriorly placed crestal portion of the graft (dashed arrows).

A kickplate functions as a dynamized implant, permitting subsidence while theoretically preventing graft dislodgment (TJ O’Brien, CSRS, 1996). Unfortunately, a kickplate resists angular deformation poorly.

\section{REGION-SPECIFIC STRATEGIES}

\section{Cervical Spine Constructs}

Historically, bone was the first interbody implant used in the cervical spine; today, it remains the most common graft material. A tricortical iliac crest graft is often used as a spacer or strut following anterior discectomy or corpectomy. Authors have disagreed on the optimal placement of the crestal portion of the graft. Proponents of anterior positioning believe that maximizing the amount of cortical bone in the anterior column of Denis provides the greatest protection against kyphosis (Fig. 1 left). This position also makes use of the "boundary effect," which predicts the greater axial load-bearing capability associated with abutment against, or gripping of, the wall of an inhomogeneous structure with its intrinsic greater density and integrity over its surface. Posterior positioning, in line with the middle column of Denis, favors axial load-bearing capability over the prevention of kyphosis (Fig. 1 center). From a biomechanical standpoint, optimal placement depends on the presence of any adjuncts to stability. If no other implant is used, anterior placement permits load sharing between the anterior column (interbody strut) and an intact posterior column (for example, the facet joints). The use of an anterior cantilevered implant permits load sharing between the middle column of Denis and the anteriorly placed implant during fusion (Fig. 1 right) (RW Murphy, CSRS, 1998).

Other bone grafts have become popular as well. The most common is the fibular allograft strut graft. Unfortunately, its high modulus of elasticity compared with the native VB bone encourages subsidence. Because of its inherently high ratio of cortical to cancellous bone, healing is negatively affected. This phenomenon may be at least partially offset by packing the core with autologous medullary bone, thus combining enhanced bone-healing capacity with the intrinsic structural strength of the fibular allograft.

Long bone strut applications present a unique constellation of decision-making dilemmas. Iliac crest grafts often have a significant curvature. This may be overcome by carefully selecting the site at which bone will be harvested and by meticulously contouring the graft (Fig. 2). Ensuring a snug fit in the VB trough reduces the incidence of graft fracture or dislodgment. In addition, the proximity of the retained VB walls aids in vascularization and ingrowth of bone from the side, thus increasing the fusion rate

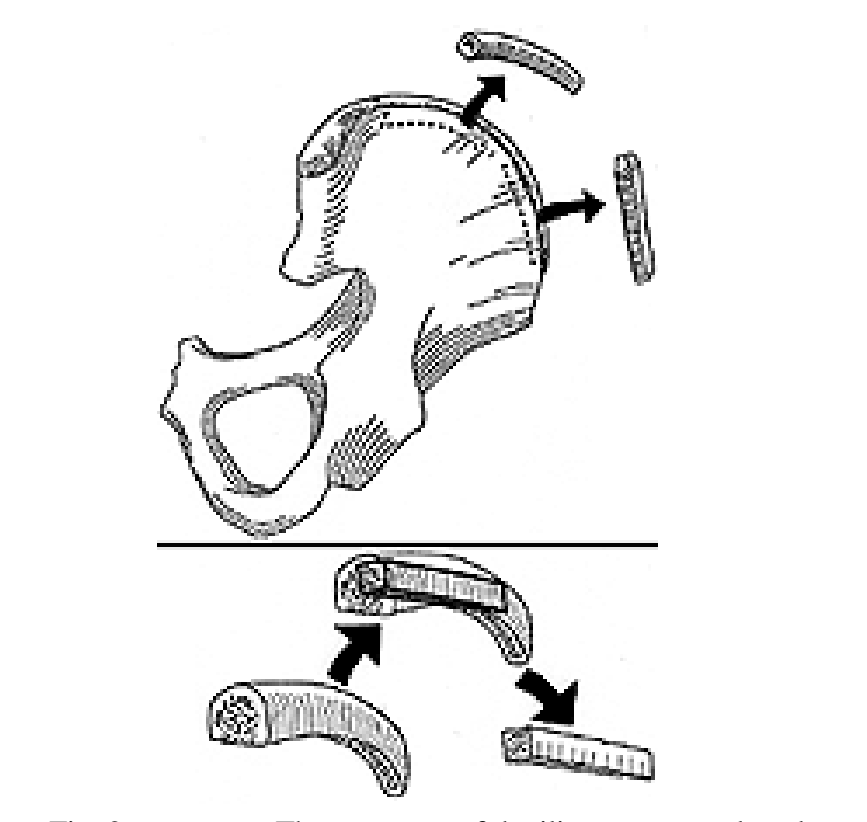

Fig. 2. Upper: The curvature of the iliac crest must be taken into consideration during graft harvesting. For long struts, a portion of the crest with a lesser curve should be selected. Lower: Shaping a curved graft in all planes allows for a close fit laterally in the case of an anterior cervical fusion (arrows). It also can minimize anterior prominence; however, care must be taken to minimize cortical bone removal. 


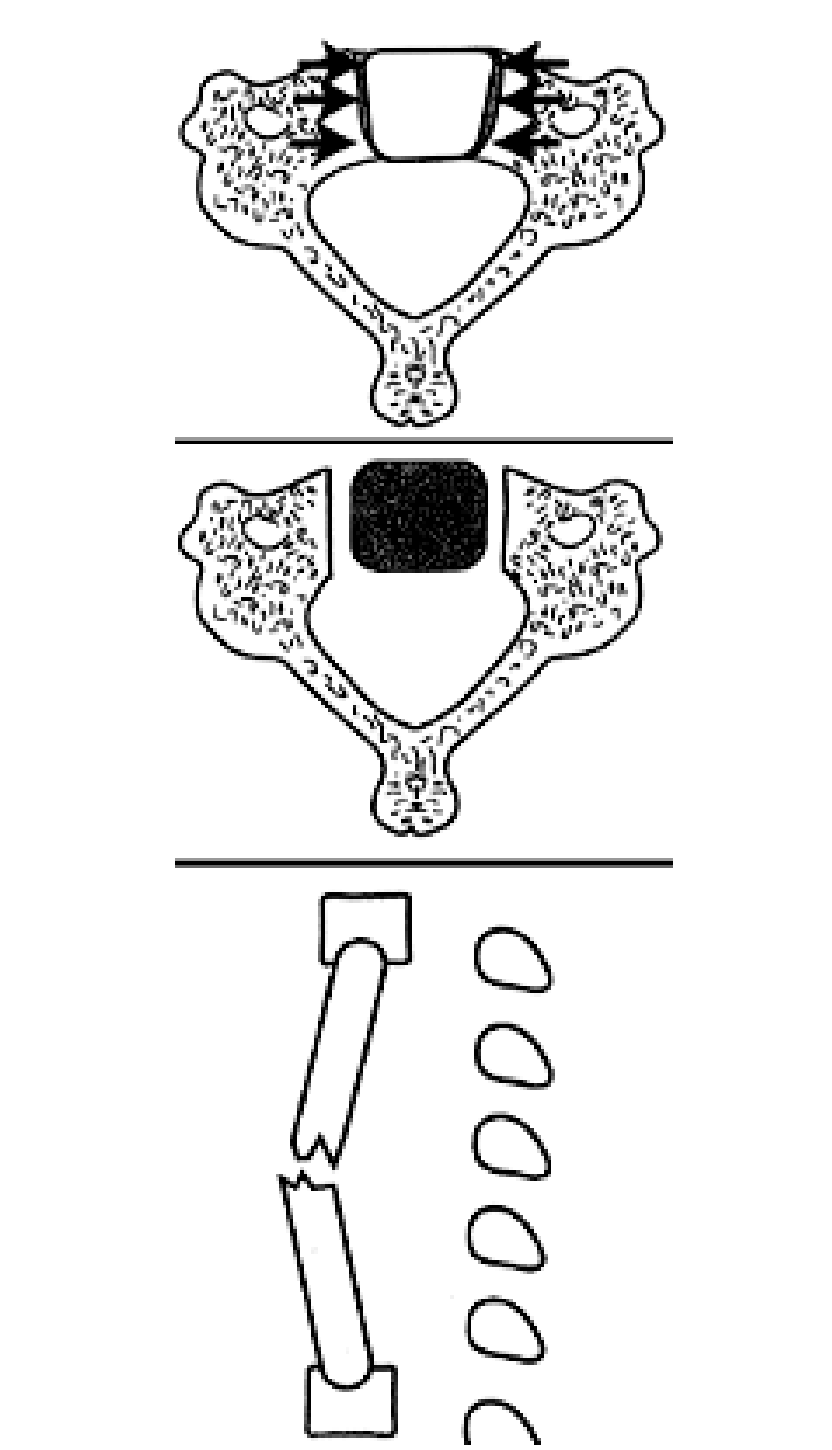

Fig. 3. Upper: Axial view of a snugly fit graft in an anterior cervical trough. This provides significant stability (arrows), as well as an increased chance for vascularization at intermediate segmental levels. Center: Because fibular struts are usually suspended in a trough, as depicted, neither of the advantages depicted in Fig. 3 upper is realized. Lower: The midportion of a graft depicted in Fig. 3 center is the most likely point of fracture.

(Fig. 3 upper). Diameter and geometrical discrepancies preclude fibular struts from being used in the same manner (Fig. 3 center). Long bone struts, particularly fibula, may occasionally fracture, most likely at the nadir of the bone's integrity due to resorption (at 4-12 months postoperatively). Beyond this window, bone integrity is augmented by vascularization, remodeling, and healing in the middle portion of the strut. Because the healing process begins at the two ends of the graft moving inward, the middle portion is the last to be structurally reconstituted; thus, it is the most prone to fracture, even when adequate fusion has occurred at the termini (Fig. 3 lower). A history of smoking is a factor that further adversely affects fibular strut graft fusion rates (NA Lowen, CSRS, 1998).

Both flat-faced and round-faced interbody fusion cages

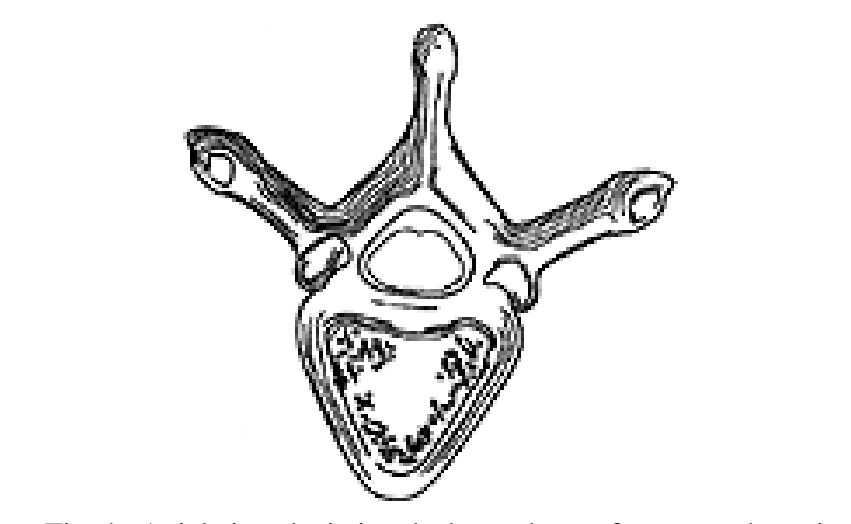

Fig. 4. Axial view depicting the heart shape of an upper thoracic vertebra.

(for example, TIFCs) are increasingly being used in the cervical spine. Flat-faced cages may be particularly useful after single- or multilevel corpectomies (JJ Abitbol, NASS, 1997). ${ }^{14}$ Wilke, et al.,${ }^{30}$ demonstrated in a human biomechanical study that round-faced cages tended to subside to a greater degree than their flat-faced counterparts. Motion does not appear to affect clinical results. ${ }^{19}$

The addition of anterior implants, in the setting of long ventral cervical struts, does not necessarily positively affect overall construct integrity (YR Rampersaud and KD Riew, CSRS, 1998). Additionally, the achievement of a solid arthrodesis may have severe adverse consequences for the adjacent native motion segments. An increase in shear strain at adjacent segments has been documented. ${ }^{21}$

\section{Thoracic Spine Constructs}

In general, the thoracic spine does not present extraordinarily unique problems for the placement of hardware. Its heart-shaped configuration in the axial plane, however, must be addressed prior to implant application (Fig. 4). Anterior surgical exposures are usually relatively difficult in this region; therefore, the number of noninterbody and nonbone strategies has increased. They include nonoperative strategies, ${ }^{8}$ interbody acrylic applications, ${ }^{11,12}$ and percutaneous vertebroplasty. ${ }^{16}$

\section{Lumbar Spine Constructs}

In the lumbar spine, interbody fusion techniques have several theoretical and proven advantages over posterior onlay grafting techniques, including: 1) a decreased incidence of pseudarthrosis; 2) an accelerated rate of fusion; and 3) an increased axial load-bearing ability.

The lumbar spine, however, can provide a hostile environment for successful bone fusion as it bears substantial axial, torsional, and translational loads. Translational loads are particularly relevant at the lumbosacral junction where the local anatomy acts to convert axial loads into translational and angular resultant force vectors (Fig. 5). Furthermore, it is difficult to resect a disc completely or to perform a complete corpectomy. On one hand, aggressive endplate debridement may result in the loss of integrity of this vital component of the axial load- bearing complex; on the other hand, incomplete debridement may result in pseudarthrosis due to inadequate preparation of the graft bed. 


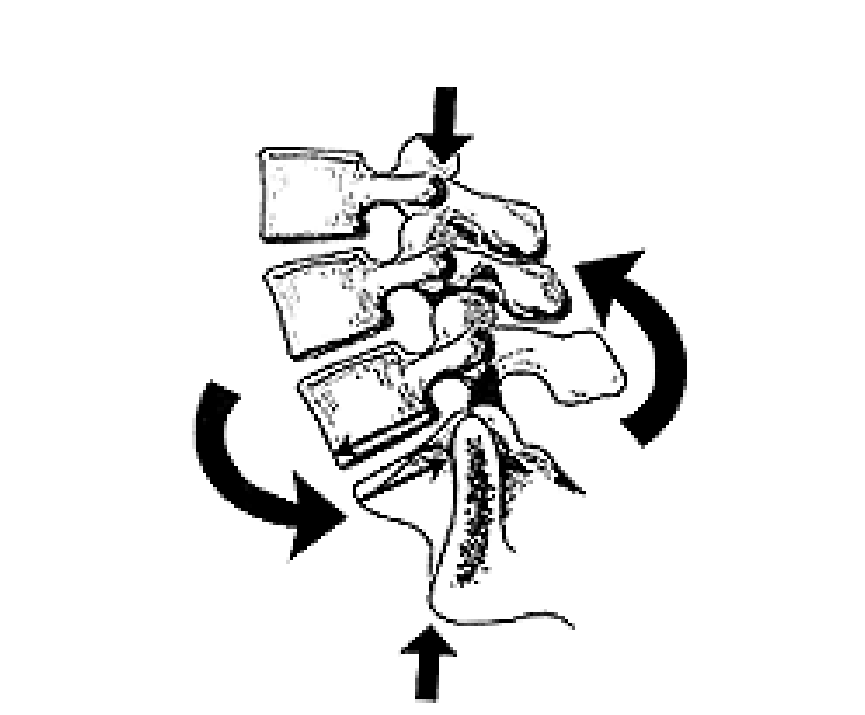

Fig. 5. The loads borne by the lumbosacral region include axial loads (vertical arrows) and resultant translational loads (diagonal arrows), as well as bending moments (curved arrows).

Posterior Lumbar Interbody Fusion. First popularized by Cloward, ${ }^{7}$ PLIF provides a technique by which to prepare the disc space and achieve an interbody fusion via a posterior approach. He emphasized careful endplate preparation, meticulous graft preparation, and the attainment of a high surface area of contact between the endplate and the bone graft (Fig. 6). He was able to achieve excellent results by using bone as his only graft material. Unfortunately, others have been unable to duplicate his success, resulting in the provision of other adjunctive procedures, such as pedicle screw fixation, to increase the clinical results of PLIF. ${ }^{12,18}$ Nonetheless, much can be gleaned from the techniques of Cloward. Above all, subsidence is governed by the cross-sectional area of contact and the relationship of the strut to the margin of the endplate. ${ }^{1}$ In newer methods of PLIF the authors have modified both the mechanical (structural support) and biological functions (bone graft for fusion) of the traditional PLIF concept by making use of rectangular cages that increase the

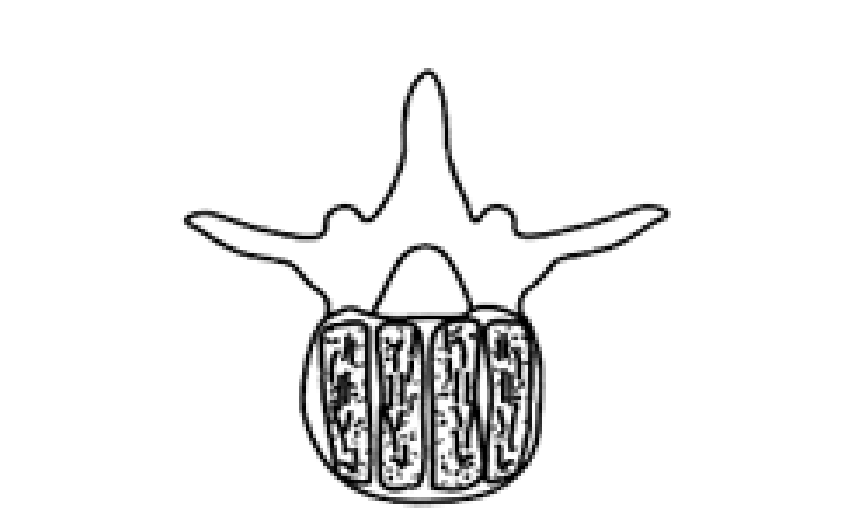

Fig. 6. When a PLIF is performed, significant surface area of contact (approximately 80\%) is achieved by placing three or four bone grafts in the interbody region. Theoretically this enhances the fusion rate, because subsidence (a negative factor in lumbar interbody fusion) is inversely proportional to the cross-sectional areas of contact.
R. J. Kowalski, L. A. Ferrara, and E. C. Benzel

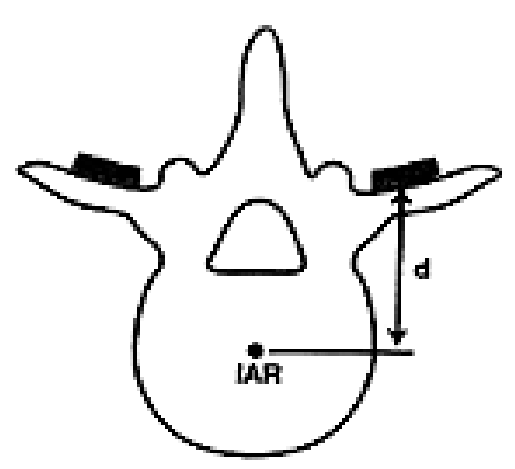

Fig. 7. The distance (d) from a lateral intertransverse fusion (solid region) to the instantaneous axis of rotation (IAR) is shown.

surface area of contact with bone, while providing structural support through the cage. ${ }^{4}$

Anterior Lumbar Interbody Fusion. Difficulties with PLIF $^{28}$ and the development of laparoscopic techniques ${ }^{20,31}$ have led to a rise in the popularity of anterior lumbar interbody fusion techniques. Here too, approach-related complications and similar pseudarthrosis rates have led others likewise to add posterior spinal implants (for example, pedicle screw fixation). ${ }^{13}$ Allograft, in which either fibula or femoral ring is used, seems to perform particularly well in this application. ${ }^{6}$

Lateral Intertransverse Fusion. With a lateral intertransverse fusion, the fusion mass is situated at a significant distance from the instantaneous axis of rotation and the neutral axis (Fig. 7). Even when a solid fusion is achieved, the resulting flexibility that persists can often result in clinical failure. ${ }^{2}$ Furthermore, the obligatory lateral soft-tissue retraction and associated soft-tissue injury may be a causative factor in the loss of lumbar lordosis and chronic back pain.

Flat-Faced Fusion Cages. Although their shapes in other planes may be varied, all flat-faced cages share the common attribute of presenting a flat surface to the accepting fusion bed (for example, the endplate region). Metal alloy cages have a much higher modulus of elasticity compared with that of bone, with carbon fiber cages and femoral ring allografts exhibiting a more intermediate value. The latter, by more closely matching the modulus of the endplate, reduces the chance of subsidence.

Although the surface area of contact varies among the different cages, it is almost always greater than that of the round-faced cages (Fig. 8) (JW Brantigan, NASS, 1997);

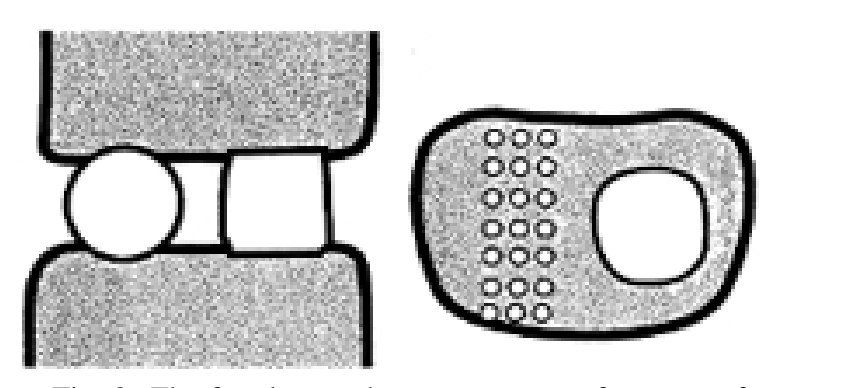

Fig. 8. Flat-faced cages have a greater surface area of contact with the VB than round-faced TIFCs. 


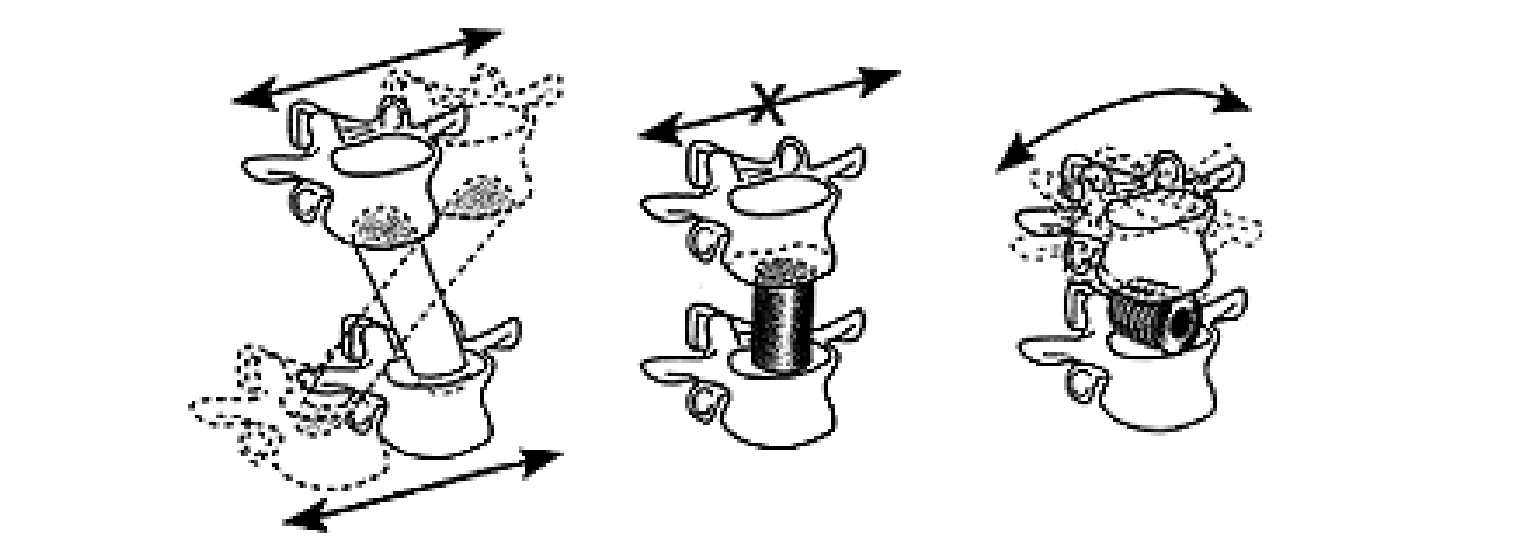

Fig. 9. Translational and angular deformation (rocking) is much more likely to occur when a strut graft with round ends is used than a flat-faced cage. A strut with a round end (left), flat-faced cage (center), and a TIFC (right) provide different resistances to rocking.

however, the greater the area of contact, even with allograft cages, the less area there is for autologous bone contact. The bone mineral density of the endplate region also has significant biomechanical implications (HS An, NASS, 1997). Compared with an interbody strut or a round-faced cage, a flat-faced cage provides a significant advantage regarding angular deformation (Fig. 9).

Round-Faced Cages. Originally developed by Bagby for application in equines (GW Bagby, NASS, 1997), TIFCs provided a method by which lumbar interbody fusion could be readily achieved, either with allograft dowels or metallic cages (GW Bagby and SD Kuslich, NASS, 1997). ${ }^{15,23,24}$ Threaded interbody fusion cages became popular, in part due to their ability to stiffen a motion segment acutely and their relative ease of application. They may even be inserted using minimally invasive techniques. ${ }^{22}$

Although there have been studies in which persistent good results have been demonstrated, ${ }^{17}$ TIFCs have had their critics. Some have questioned the use of very young- age study populations (mean 41.5 [HA Yuan, unpublished data] and $42.1^{24}$ years of age), the definition of a solid fusion (less than $5^{\circ}$ of motion in the sagittal plane), and the absence of lucency surrounding the cage. ${ }^{24}$ Biomechanical concerns include the possible sequelae of disrupting the anterior and posterior ligaments during insertion (NM Grosland, NASS, 1997) and the cage's ability to withstand loading in shear manifest in the L5-S1 disc space once a patient assumes an upright posture (Fig. 5).

With these cages a round face is presented to the fusion bed. As indicated by Cloward, ${ }^{7}$ maximizing the surface area of contact is critical. The degree of bone-on-bone contact varies among the clinically available TIFCs. For a given cage, placement in a lordotic spine may vary the quality of bone-on-bone contact throughout the length of the cage (Fig. 10). ${ }^{3}$ Finally, the observation that explanted human cages have contained predominantly collagen and woven bone raises doubts about the integrity and quality of intracage bone (B Cunningham, NASS, 1999).

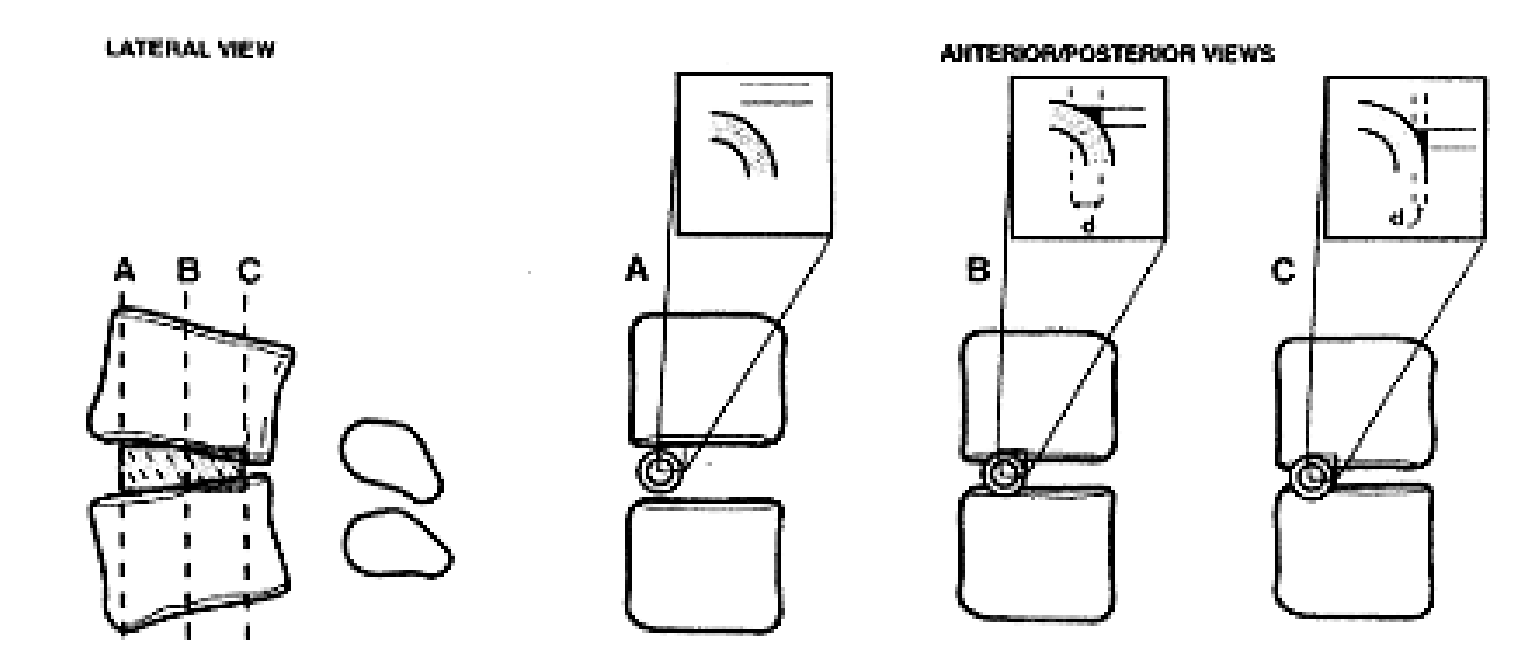

Fig. 10. Depiction of a TIFC placed "between" two nonparallel endplates (lordosis present); lateral view (left), and three coronal sections (A, B, and C; right). Note the variation of penetration of the cage into the endplates (A, B, and C). The enlarged diagram depicts the region of contact (black triangles, of width "d") between the surface of the cage and the endplate. In this lordotic configuration, the region of contact varies throughout the length of the implant. This variation may adversely affect stability and result in a nonuniform penetration of the implant into the adjacent VBs. 


\section{SUBSIDENCE-RELATED BIOMECHANICS}

\section{Bone Shaping and Fitting}

The ultimate outcome of a surgical procedure hinges on several factors. Although an implant may fail of its own accord, more commonly failure may be directly or indirectly attributed to the surgeon. A surgeon may choose the wrong operation or the wrong implant, or fail to use proper techniques. We have attempted to provide a basic theoretical knowledge of the relevant biomechanics, as well as clinical spine region-specific applications. This should help with choosing the right operation and the best implant/construct. We now turn our attention to those intraoperative techniques that can literally make or break a fusion.

Good "carpentry" of bone components is critical for optimizing bone fusion outcome. The creation and shaping of a mortise in the VB is equally as important as the precise fitting and shaping of an interbody bone graft in minimizing the chance of dislodgment and other forms of failure. Three factors directly affect the incidence and extent of subsidence: 1) the closeness of fit of the bone graft in the VB mortise; 2) the surface area of contact between the bone graft and VB; and 3) the character or quality of the contact surfaces.

\section{Closeness of Fit}

Just as we learn in childhood, that square pegs do not set firmly in round holes, squared-off bone grafts do not fit well in a round mortise and vice versa (Fig. 11 upper left). A poor fit increases the likelihood of two types of adverse outcomes: 1) nonunion due to an inadequate surface area

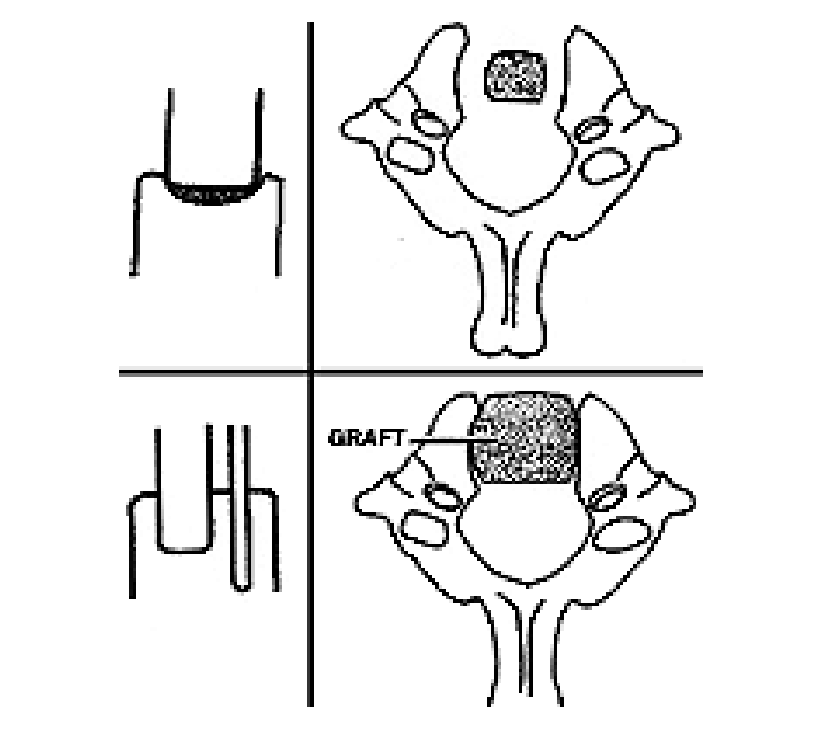

Fig. 11. Upper Left: A square (flat)-ended bone graft does not fit well into a rounded VB mortise. Thus, nonunion may occur due to an inadequate surface area of contact (shaded region). Upper Right: If the bone graft diameter is too small, the graft is suspended in space, effectively eliminating the buttressing and healing advantage. Lower Left: Smaller cross-sectional area grafts subside more than large cross-sectional area grafts. Lower Right: Maximizing the surface area of contact and the closeness of fit between the bone graft and the VB minimizes concentration of stress and the chance of nonunion or excessive subsidence.

\section{R. J. Kowalski, L. A. Ferrara, and E. C. Benzel}

of contact (Fig. 11 upper right); and 2) excessive subsidence due to the concentration of stresses and loads at the points of contact between the VB and the bone graft (Fig. 11 lower left). On the other hand, maximizing the surface area of contact and optimizing the closeness of fit between the bone graft and the VB minimize stress concentration and, hence, minimize the chance of nonunion or excessive subsidence. (Fig. 11 lower right).

\section{Surface Area of Contact}

The extent of subsidence is inversely proportional to the surface area of contact between the bone graft and the VB. The larger the surface area of contact, the less the subsidence occurs, and vice versa. A basic example illustrates this principle: the force required to penetrate a Styrofoam block with the eraser end of a pencil is much greater than that required for the sharpened end.

\section{Quality of the Contact Surfaces}

Two qualities determine the efficacy of the contact surfaces: 1) the extent of endplate preservation; and 2) the proximity of the point of contact to the edge of the VB. Emery, et al. ${ }^{10}$ have observed that simply burring the endplate results in higher fusion rates without increasing the degree of clinically significant settling. Anyone who has stood on an aluminum soda can has realized the power of the "boundary effect." The VB cortex is superior to the softer inner cancellous bone at bearing axial loads. The size of the load that may be borne when all or a portion of the load is placed over the VB margin is dramatically increased by the buttressing effect of the cortical boundary. ${ }^{29}$ Thus, the greatest biomechanical advantage is achieved with a bone graft that makes contact with the entire VB surface and is the same size, making contact with all of the cortical margin.

\section{CONCLUSIONS}

Bone fusion involves the successful integration of several factors. First, one must understand the relevant biomechanical theory including the concept of bone as an implant. Second, one must be familiar with the variety of available constructs and the appropriate techniques required to apply them. Here, we have tried to describe these in a region-specific manner. Third, one must be proficient at the intraoperative skills that may make the difference between successful fusion and pseudarthrosis.

\section{References}

1. Bagby GW: Arthrodesis by the distraction-compression method using a stainless steel implant. Orthopedics 11:931-944, 1988

2. Barrick WT, Schofferman JA, Reynolds JB, et al: Anterior lumbar fusion improves discogenic pain at levels of prior posterolateral fusion. Spine 25:853-857, 2000

3. Benzel EC, Ferrara L, Baldwin N: Multidirectional stabilizing potential of BAK interbody spinal fusion system for anterior surgery. J Spinal Disord 11:454-455, 1998

4. Brantigan JW, Steffee AD, Geiger JM: A carbon fiber implant to aid interbody lumbar fusion. Mechanical testing. Spine 16 (Suppl 6):S277-S282, 1991

5. Brantigan JW, Steffee AD, Lewis ML, et al: Lumbar interbody fusion using the Brantigan $\mathrm{I} / \mathrm{F}$ cage for posterior lumbar inter- 


\section{Biomechanics of bone fusion}

body fusion and the variable pedicle screw placement system: two-year results from a Food and Drug Administration investigational device exemption clinical trial. Spine 25:1437-1446, 2000

6. Buttermann GR, Glazer PA, Hu SS, et al: Revision of failed lumbar fusions. A comparison of anterior autograft and allograft. Spine 22:2748-2755, 1997

7. Cloward RB: The treatment of ruptured lumbar intervertebral discs by vertebral body fusion. J Neurosurg 10:154-168, 1953

8. de Klerk LW, Fontijne WP, Stijnen T, et al: Spontaneous remodeling of the spinal canal after conservative management of thoracolumbar burst fractures. Spine 23:1057-1060, 1998

9. DeBowes RM, Grant BD, Bagby GW, et al: Cervical vertebral interbody fusion in the horse: a comparative study of bovine xenografts and autografts supported by stainless steel baskets. Am J Vet Res 45:191-199, 1984

10. Emery SE, Bolesta MJ, Banks MA, et al: Robinson anterior cervical fusion. Comparison of the standard and modified techniques. Spine 19:660-663, 1994

11. Errico TJ, Cooper PR: A new method of thoracic and lumbar body replacement for spinal tumors: technical note. Neurosurgery 32:678-681, 1993

12. Gokaslan ZL, York JE, Walsh GL, et al: Transthoracic vertebrectomy for metastatic spinal tumors. J Neurosurg 89: 599-609, 1998

13. Hacker RJ: Comparison of interbody fusion approaches for disabling low back pain. Spine 22:660-666, 1997

14. Hacker RJ: A randomized prospective study of an anterior cervical interbody fusion device with a minimum of 2 years of follow-up results. J Neurosurg 93 (Suppl 2):222-226, 2000

15. Heim SE, Altimari AF, Norek MT: The treatment of lumbar degenerative motion segment pain. Spinal Frontiers June:6-8, 1997

16. Jensen ME, Evans AJ, Mathis JM, et al: Percutaneous polymethylmethacrylate vertebroplasty in the treatment of osteoporotic vertebral body compression fractures: technical aspects. AJNR 18:1897-1904, 1997

17. Kuslich SD, Danielson G, Dowdle JD, et al: Four-year followup results of lumbar spine arthrodesis using the Bagby and Kuslich lumbar fusion cage. Spine 25:2656-2662, 2000

18. Lin PM, Cautilli RA, Joyce MF: Posterior lumbar interbody fusion. Clin Orthop 180:154-165, 1983

19. Lofgren H, Johannsson V, Olsson T, et al: Rigid fusion after Cloward operation for cervical disc disease using autograft, allograft, or xenograft: a randomized study with radiostereometric and clinical follow-up assessment. Spine 25:1908-1916, 2000
20. Mathews HH, Evans MT, Molligan HJ, et al: Laparoscopic discectomy with anterior lumbar interbody fusion. A preliminary review. Spine 20:1797-1802, 1995

21. Matsunaga S, Kabayama S, Yamamoto T, et al: Strain on intervertebral discs after anterior cervical decompression and fusion. Spine 24:670-675, 1999

22. McAfee PC, Regan JJ, Geis WP, et al: Minimally invasive anterior retroperitoneal approach to the lumbar spine. Emphasis on the lateral BAK. Spine 23:1476-1484, 1998

23. Onesti ST, Ashkenazi E: The Ray threaded fusion cage for posterior lumbar interbody fusion. Neurosurgery 42:200-205, 1998

24. Ray CD: Threaded titanium cages for lumbar interbody fusions. Spine 22:667-680, 1997

25. Saunders RL: On the pathogenesis of the radioculopathy complicating multilevel corpectomy. Neurosurgery 37:408-413, 1995

26. Saunders RL, Bernini PM, Shirreffs TG, et al: Central corpectomy for cervical spondylotic myelopathy: a consecutive series with long-term follow-up evaluation. J Neurosurg 74: 163-170, 1991

27. Saunders RL, Pikus HJ, Ball P: Four-level cervical corpectomy. Spine 23:2455-2461, 1998

28. Verlooy J, De Smedt K, Selosse P: Failure of a modified posterior lumbar interbody fusion technique to produce adequate pain relief in isthmic spondylolytic grade 1 spondylolisthesis patients. A prospective study of 20 patients. Spine 18: 1491-1495, 1993

29. Wang JC, Zou D, Yuan H, et al: A biomechanical evaluation of graft loading characteristics for anterior cervical discectomy and fusion. A comparison of traditional and reverse grafting techniques. Spine 23:2450-2454, 1998

30. Wilke HJ, Kettler A, Goetz C, et al: Subsidence resulting from simulated postoperative neck movements: an in vitro investigation with a new cervical fusion cage. Spine 25:2762-2770, 2000

31. Zucherman JF, Zdeblick TA, Bailey SA, et al: Instrumented laparoscopic spinal fusion. Preliminary results. Spine 20: 2029-2035, 1995

Manuscript received February 27, 2001.

Accepted in final form March 20, 2001.

Address reprint requests to: Edward C. Benzel, M.D., F.A.C.S., Spinal Disorders, Cleveland Clinic Foundation, Department of Neurosurgery/S80, 9500 Euclid Avenue, Cleveland, Ohio 44195. email: benzele@ccf.org. 